\title{
Stage IIIA Mycosis Fungoides and Sezary Syndrome AJCC v8
}

National Cancer Institute

\section{Source}

National Cancer Institute. Stage IIIA Mycosis Fungoides and Sezary Syndrome A/CC v8. NCl Thesaurus. Code C141354.

Stage IIIA includes: T4, N0-2, M0, B0. T4: Confluence of erythema covering 80\% or more of body surface area. N0: No clinically abnormal peripheral lymph nodes; biopsy not required. N1: Clinically abnormal peripheral lymph nodes; histopathology Dutch grade 1 or National Cancer Institute (NCI) LNO-2. N2: Clinically abnormal peripheral lymph nodes; histopathology Dutch grade 2 or NCI LN3. M0: No visceral org an involvement. B0: Absence of significant blood involvement: $5 \%$ or less of peripheral blood lymphocytes are atypical (Sezary cells). (AJCC 8th ed.) 